\title{
Strong and Auxiliary Forms of the Semi-Lagrangian Method for Incompressible Flows
}

\author{
D. Xiu ${ }^{1}$, S.J. Sherwin ${ }^{2}$, S. Dong ${ }^{1}$ and G.E. Karniadakis ${ }^{1 *}$ \\ ${ }^{1}$ Division of Applied Mathematics, Brown University \\ ${ }^{2}$ Department of Aeronautics, Imperial College
}

\begin{abstract}
We present a review of the semi-Lagrangian method for advection-diffusion and incompressible Navier-Stokes equations discretized with high-order methods. In particular, we compare the strong form where the departure points are computed directly via backwards integration with the auxiliary form where an auxiliary advection equation is solved instead; the latter is also referred to as Operator Integration Factor Splitting (OIFS) scheme. For intermediate size of time steps the auxiliary form is preferrable but for large time steps only the strong form is stable.
\end{abstract}

${ }^{*}$ Corresponding author, gk@dam.brown.edu 


\section{Introduction}

The Semi-Lagrangian method has been used primarily in advection-diffusion systems due to its two useful attributes: (1) large time step allowed, and (2) stability. Moreover, implementing it with high-order discretizations leads to minimum dispersion error. The semi-Lagrangian approach has long been used in meteorology for numerical weather prediction, where the use of large time step is essential for efficiency [1]. Its use, however, in Navier-Stokes simulations has been sporadic but recent work has demonstrated its efficiency, especially in the context of high Reynolds number simulations $[2,3]$. In this review paper, we present the two main versions of the semi-Lagrangian method and examine several issues related to convergence, efficiency, stability and ease of implementation.

This method was introduced at the beginning of the 1980s [4, 5], and the basic idea is to discretize the Lagrangian derivative of the solution in time instead of the Eulerian derivative. As we shall see in the next section this requires the solution at the foot of the characteristic from each discrete mesh point. The solution at the characteristic foot can be determined either by using backward particle tracking or equivalently by solving an auxiliary advection equation. The first version of the method is often referred to as the strong form. The second scheme is sometimes refereed to as the Operator Integration Factor Splitting (OIFS) scheme [2]. However, as we shall demonstrate it can also be interpreted more as a semi-Lagrangian scheme with an auxiliary advection equation; therefore, we shall refer to it as the auxiliary semi-Lagrangian scheme. Further details about different forms of strong and weak forms of semi-Lagrangian schemes can be found in [6] and references therein.

We now examine the potential effectiveness of the method in the context of direct numerical simulation of turbulent flows (DNS) at high Reynolds number $(R e)$. Simple estimates based on the Kolmogorov dissipative length scale suggest that the required number of degrees of freedom scale as $R e^{9 / 4}$ in three-dimensions [7]. What is not factored, however, in such estimate is the computational cost associated with the time-integration of the Navier-Stokes equations, which in practice, may be the prohibitive factor. After all, in a parallel computation the spatial resolution requirements can be alleviated by domain decomposition whereas the time-stepping cost cannot be avoided.

To illustrate the current inefficiency of time-discretization, let us consider the often-used semi-implicit Eulerian scheme, where advection is treated explicitly. The maximum allowable time step is dictated by the CFL number. In [3] the following ratio between the CFL-dictated time step $\Delta t_{C F L}$ and the Kolmogorov temporal scale $\tau$ has been derived

$$
\frac{\Delta t_{C F L}}{\tau} \propto R e^{1 / 2} \frac{1}{N^{\alpha}} \propto R e^{1 / 2-3 \alpha / 4}
$$

Here, $N^{\alpha}$ represents the scaling of the maximum eigenvalue associated with the spectral discretization, with $N$ the 
total number of nodes in one dimension. For example, for a Fourier discretization $\alpha=1$; for Chebyshev discretization (used often in DNS of wall-bounded turbulence) $\alpha=2$; and for spectral $/ h p$ element methods $\alpha \approx 3 / 2$ (see [8], ch. 6). It is clear from equation (1) that at Reynolds number of 10,000, the maximum allowable time step can be one order $(\alpha=1)$ to four orders $(\alpha=2)$ of magnitude smaller than the temporal Kolmogorov scale. Therefore, in most spectral DNS of inhomogeneous turbulence (where $\alpha \geq 3 / 2$ ) there is an uneven distribution of resolution in space and time, with the smallest spatial scale approximately matched but with the temporal scale over-resolved by at least two to three orders of magnitude. This inefficiency of currently employed semi-implicit schemes for DNS of inhomogeneous turbulence has been recognized before, and attempts have been made to employ fully implicit schemes. However, this requires Newton iterations and non-symmetric solvers that render the overall approach inefficient. Progress can be made by employing semi-Lagrangian time-discretization, which could increase significantly the maximum allowable time step while maintaining the efficiency of symmetric solvers.

The semi-Lagrangian method depends strongly on the spatial discretization. Specifically, its accuracy is particularly sensitive to the method of backward integration of the characteristic equation as well as the interpolation scheme to evaluate the solution at departure points. This has been shown by Falcone \& Ferretti [9] who conducted a rigorous analysis of the stability and convergence properties of semi-Lagrangian schemes. Typically, the backward integration is performed by employing second-order schemes (i.e., mid-point rule), explicitly or implicitly. In [10] and [11], the fourth-order Runge-Kutta method was employed but their results did not show any improvement over the second-order schemes. This finding is perhaps due to low spatial resolution used in these works, which is crucial for the overall accuracy of semi-Lagrangian schemes. The simplest semi-Lagrangian scheme with linear interpolation is equivalent to the classical first-order upwinding scheme [12], which is excessively dissipative (see [4] and [13]). A popular and effective choice for interpolation methods in previous works has been the cubic spline methods [14]; see also [15].

The importance of high-order discretization in semi-Lagrangian methods was demonstrated by Giraldo who analyzed the dispersion and diffusion errors of the one-dimensional linear advection equation [16]. He employed the standard Eulerian spectral element formulation and a semi-Lagrangian formulation. Giraldo showed conclusively that the addition of the semi-Lagrangian scheme to the spectral element method introduces a proper dissipation mechanism that the method requires for high values of the Courant number. In particular, for linear elements the dispersion errors are plotted in figure 1 for both discretizations. At this order, both schemes suffer from dispersion errors (isocontours with values less or greater than 1) but have different error distributions. There is no error in the amplification factor, i.e. no numerical diffusion, for the standard spectral element method but the semi-Lagrangian 

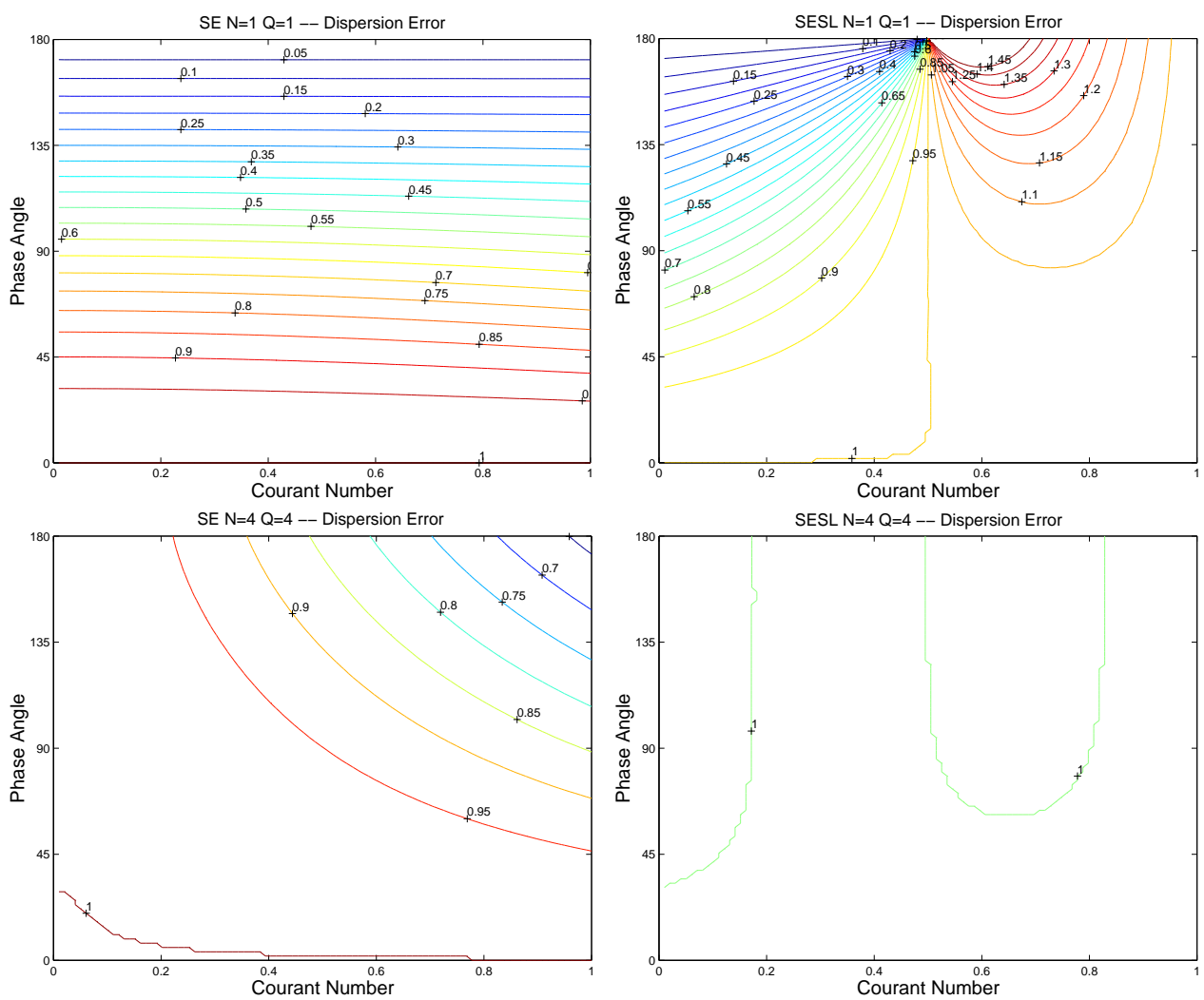

Figure 1: Upper: Order $P=1$. Isocontours of phase errors for different frequencies (vertical axis) and Courant number (horizontal axis). Left: Eulerian spectral element scheme. Right: semi-Lagrangian scheme. Lower: Order $P=4$. (Courtesy of F.X. Giraldo [16])

scheme exhibits appreciable numerical diffusion especially around Courant number $C=0.5$ and high frequencies. In fact, this is the region where the dispersive errors are large as well, thus acting "in concert" with diffusion. However, for spectral order greater than $P=4$ all dispersion errors are eliminated in the semi-Lagrangian scheme but not in the standard spectral element scheme as shown in figure 1. This behavior of the semi-Lagranagian scheme is true even for Courant number $C \gg 1$, see [16].

An intriguing finding is that the error of semi-Lagrangian schemes in solving advection-diffusion equations decreases as the time step increases in a certain range of parameters, and this has initially led to some erroneous justifications $[17,18]$. The error analysis in [9] showed that the overall error of semi-Lagrangian method is indeed not monotonic with respect to time step $\Delta t$, and, in particular, it has the form

$$
\mathcal{O}\left(\Delta t^{k}+\frac{\Delta x^{P+1}}{\Delta t}\right),
$$

where $k$ refers to the order of backward time integration and $P$ to the interpolation order; similar conclusions had been reached earlier in [19]. 
The extension of semi-Lagrangian method to the solution of Navier-Stokes equations was presented in the pioneering work of Pironneau [5]. He demonstrated nonlinear stability of the method even as the viscosity approaches to zero. He also obtained suboptimal error estimates, which were improved later by Süli [20]. Most of the previous analysis and numerical implementations in CFD applications have employed the Taylor-Hood finite element and are first-order in time $[21,10,22]$. In a more recent paper [23], an error analysis was conducted for the fractional-step method for incompressible Navier-Stokes equations. In particular, the pressure-correction version of the fractional scheme with first-order time-stepping was analyzed and an extension to a second-order was proposed but not analyzed. An attempt at a second-order scheme was made in [21] but no convergence rates were documented in that work. Moreover, results presented for the standard benchmark problem of driven-cavity flow are markedly different than accepted results in the literature, possibly due to an erroneous treatment of the pressure term.

In this paper, we present the strong and auxiliary forms of the semi-Lagrangian method applied to the NavierStokes equations. First, we explain the method in the context of a scalar advection-diffusion equation in the next section and demonstrate its convergence properties. We then present the discretization for the incompressible NavierStokes equations following a spectral/hp element method as well as a mixed spectral/spectral element method, as the latter is used often in simulations of inhomogeneous turbulent flows in simple geometries. Here we also discuss details of the parallel implementation as the semi-Lagrangian method is typically more difficult to implement in parallel than the Eulerian approach. We conclude the paper with a discussion of relative merits and open issues of the two formulations.

\section{Advection-Diffusion Equation}

\section{$2.1 \quad$ Formulation}

In this section, we review the strong and auxiliary forms of the semi-Lagrangian method for advection-diffusion equations. We first present the main ideas and subsequently we present benchmark results. We employ a spectral $/ h p$ element discretization and examine the fast convergence of the combined schemes.

To demonstrate these two approaches we can consider the scalar advection-diffusion equation

$$
\frac{\partial \phi}{\partial t}+\mathbf{a} \cdot \nabla \phi=\nu \nabla^{2} \phi
$$

where $\mathbf{a}(\mathbf{x}, t)$ is an advection field. Equation (2) can equivalently be written in Lagrangian form as

$$
\frac{D \phi}{D t}=\nu \nabla^{2} \phi \quad \text { with } \quad \frac{D}{D t}=\frac{\partial}{\partial t}+\mathbf{a} \cdot \nabla
$$


a)

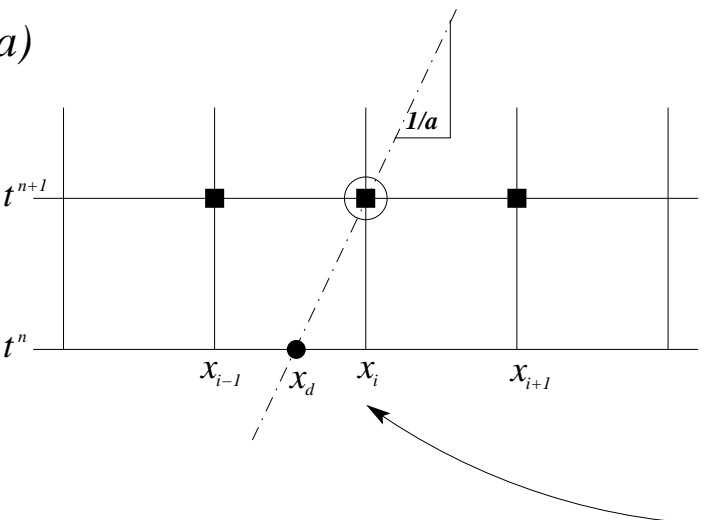

b)

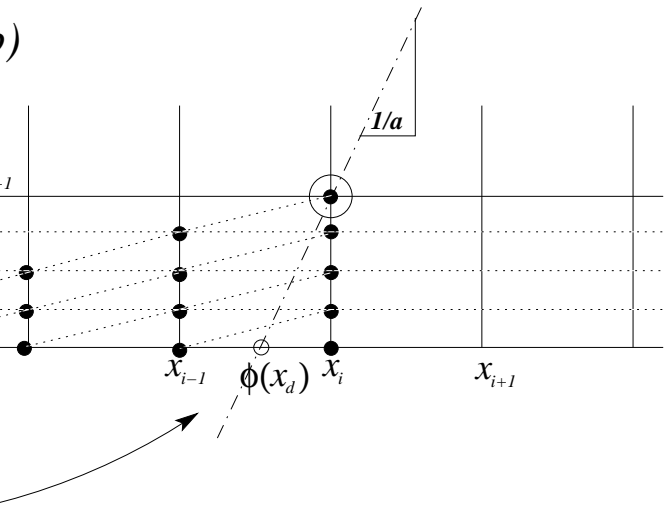

Figure 2: a) Schematic of the strong semi-Lagrangian approximation where the particle is backward tracked from $x_{i}^{n+1}$ to determine $x_{d}$ and $\phi\left(x_{d}\right)$. b) Schematic representation of the auxiliary semi-Lagrangian approximation where a hyperbolic advection equation is advanced using a smaller time step to determine $\tilde{\phi}\left(\boldsymbol{x}_{i}, t^{n+1}\right)$;

where $D / D t$ is the Lagrangian or material derivative moving with the advection velocity i.e., $D \mathbf{x} / D t=\mathbf{a}$. Following the semi-Lagrangian approach we can discretize equation (3) in time at point $x_{i}$ using a first-order implicit scheme to obtain

$$
\frac{\phi_{i}^{n+1}-\phi_{d}^{n}}{\Delta t}=\nu \nabla^{2} \phi_{i}^{n+1},
$$

where $\phi_{d}^{n}=\phi^{n}\left(\mathbf{x}_{d}, t^{n}\right)$ and $\mathbf{x}_{d}$ is the so-called departure point.

In the strong form the material derivative is evaluated along the characteristics; we can determine the departure point $\mathbf{x}_{d}$ by solving the characteristic equation $D \mathbf{x} / D t=\mathbf{a}$ backward in time from $t^{n+1} \geq t \geq t^{n}$ using the initial conditions $\mathbf{x}\left(t^{n+1}\right)=\mathbf{x}_{i}$. This approach is schematically represented in figure 2(a) where we show the onedimensional discretization on a discrete $x-t$ diagram. The implicit discretization of the diffusion term is represented by the black squares. Subsequently, $\phi\left(\mathbf{x}_{d}\right)$ is evaluated and the approximation to the Lagrangian time derivative can then be determined. The complexity of backward particle tracking depends on the form of $\mathbf{a}(\mathbf{x}, t)$. If $\mathbf{a}(\mathbf{x}, t)$ is time independent the calculation is relatively straight-forward on a well behaved discretization. However, if the convection velocity is time dependent then different time integration strategies can be adopted, see [3, 16]. We also note that this scheme requires an interpolation operation to evaluate $\phi\left(\mathbf{x}_{d}, t^{n}\right)$.

In the auxiliary semi-Lagrangian approach the starting point is similar to the strong semi-Lagrangian technique[2], however instead of backward particle tracking we solve for the departure solution $\phi_{d}^{n}$ directly. This can be achieved by solving the advection part of the problem independently in its Eulerian form and with a smaller time step. To this end, we introduce an intermediate solution $\tilde{\phi}(\mathbf{x}, \tau)$ and solve the problem:

$$
\frac{D \tilde{\phi}}{D \tau}=\frac{\partial \tilde{\phi}}{\partial \tau}+\mathbf{a} \cdot \nabla \tilde{\phi}=0, \quad t^{n} \leq \tau \leq t^{n+1}
$$


with initial conditions $\tilde{\phi}\left(\mathbf{x}_{i}, t^{n}\right)=\phi\left(\mathbf{x}_{i}, t^{n}\right)$. Since this is a strictly hyperbolic equation then along the characteristic the solution is constant and so $\phi\left(\mathbf{x}_{d}, t^{n}\right)=\tilde{\phi}\left(\mathbf{x}_{i}, t^{n+1}\right)$. The auxiliary semi-Lagrangian method is shown schematically in figure 2(b), where the solution at the departure point is determined by the discretization of equation (5) using a smaller time step. The explicit solution of equation (5) means that from stability considerations the time step is restricted by a CFL condition. Nevertheless, providing the solution of (5) can be solved more efficiently than the implicit diffusion operator, then equation (5) can be discretized with a time step near to the CFL limit while the implicit diffusion operator is solved less frequently in time thereby reducing the cost of the overall algorithm for a fixed integration time. Accordingly, it is the ratio of the cost of the explicit advection term to the implicit diffusion operator which ultimately limits the possible speedup. We also note that the time accuracy is governed by the larger time step applied on the implicit diffusion operator.

The explicit integration of the auxiliary advection equation (5) could be quite restrictive and this may make the solution of equation (5) relatively costly. To make this technique tractable, it requires an efficient technique to solve that equation, which has implications for the type of spatial discretization adopted. It also requires an estimate of the time step restriction for the advection problem. To this end, using a nodal spectral element Galerkin discretization with Gauss-Lobatto-Legendre quadrature points leads to a diagonal mass matrix [8]. If a modal spectral element method is used, then a discontinuous Galerkin formulation should be adopted which also leads to a diagonal mass matrix. The first approach has been adopted by a variety of researchers including [2, 24, 25], while the second approach was proposed in [26] and has also been applied to the incompressible Navier-Stokes equation as discussed in the next section.

\subsection{Convergence}

Adopting the standard benchmark problem applied in [3] we consider the advection and diffusion of a Gaussian-cone with a transport velocity field of

$$
u=+x_{2}, \quad v=-x_{1} .
$$

and initial condition of

$$
\phi\left(x_{1}, x_{2}, 0\right)=e^{-\left[\left(x_{1}-x_{1}^{0}\right)^{2}+\left(x_{2}-x_{2}^{0}\right)^{2}\right] / 2 \lambda^{2}}
$$

The exact solution to this problem is

$$
\phi\left(x_{1}, x_{2}, t\right)=\frac{\lambda^{2}}{\lambda^{2}+2 \nu t} e^{-\left[\hat{x}_{1}^{2}+\hat{x}_{2}^{2}\right] / 2\left(\lambda^{2}+2 \nu t\right)}
$$


where

$$
\hat{x_{1}}=x_{1}-x_{1}^{0} \cos t-x_{2}^{0} \sin t, \quad \hat{x_{2}}=x_{1}+x_{1}^{0} \sin t-x_{2}^{0} \cos t .
$$

Fixing the constants as $\lambda=\frac{1}{8}$; and $\left(x_{1}^{0}, x_{2}^{0}\right)=\left(-\frac{1}{2}, 0\right)$ we discretize the problem with a mesh consisting of $10 \times 10$ quadrilateral elements in the region $-1 \leq x_{1}, x_{2} \leq 1$. The solution is then time-integrated for one revolution corresponding to a final time of $t=2 \pi$.

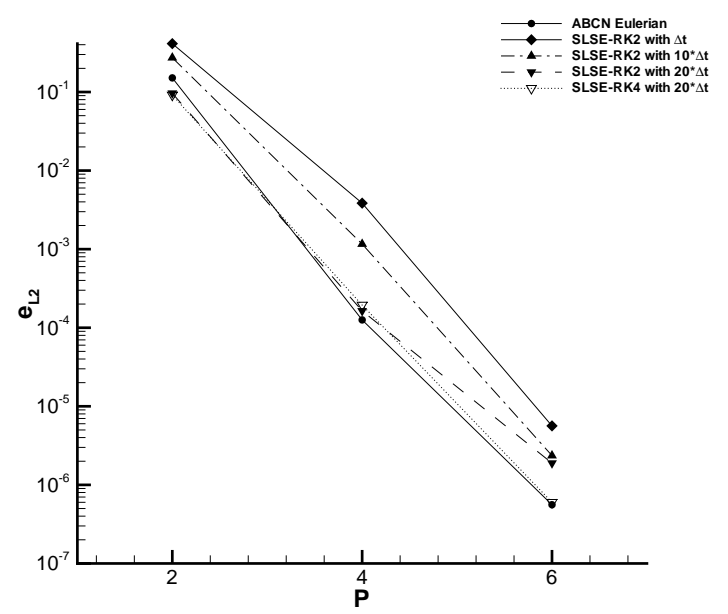

Figure 3: Spatial convergence of Eulerian and strong semi-Lagrangian methods at large $\Delta t$; Gaussian-cone problem.

In figure 3 we plot the $L_{2}$ error from an Eulerian method (Adams-Bashforth combined with Crank-Nicolson, $\mathrm{ABCN}$ ) and the strong semi-Lagrangian spectral/ $h p$ element (SLSE) method with fixed CFL number $C=0.5$ and diffusion number $D=0.01$. The backward integration is an explicit mid-point rule denoted here as RK2 method. The spectral order varies from $P=2$ to 6 . We observe on this semi-log plot that spectral convergence is achieved for both methods. The SLSE method gives relatively larger error at lower polynomial order $P$, but it quickly reaches the $\mathcal{O}\left(\Delta t^{2}\right)$ temporal error limit at $P=6$. Results with $10 \Delta t$ and $20 \Delta t$, which correspond to CFL numbers of 5 and 10 , are also plotted. We observe that as the time step increases, the error is reduced, matching the error of the Eulerian scheme but at time step size twenty times larger. Also, a further improvement with the fourth-order Runge-Kutta method (RK4) is obtained at $20 \Delta t$ with polynomial order $P=6$.

The error in the strong semi-Lagrangian method consists of two parts: the error of the backward integration $\mathcal{O}\left(\Delta t^{k+1}\right)$ and the error from interpolation $\mathcal{O}\left(\Delta x^{P+1}\right)$, where $k$ is the order of integration method and $P$ is the order of the polynomial basis. Therefore, the overall accuracy of semi-Lagrangian method is

$$
\frac{\boldsymbol{a}^{n+1}-\boldsymbol{a}_{d}^{n}}{\Delta t}=\frac{d \boldsymbol{a}}{d t}+\mathcal{O}\left(\Delta t^{k}+\frac{\mathcal{O}\left(\Delta x^{P+1}\right)}{\Delta t}\right)
$$


A rigorous derivation of the above expression can be found in [9]. Equation (6) shows that the error is not monotonic with respect to $\Delta t$. When the polynomial order $P$ is small, the interpolation error dominates. As $\Delta t$ increases, the overall error decreases. It can also be appreciated that when the first term $\mathcal{O}\left(\Delta t^{k}\right)$ is subdominant, further increasing $k$ will not improve the overall accuracy, which explains why there is no improvement with a fourth-order Runge-Kutta method over the second-order methods for low-order discretizations. On the other hand, when the spatial error is subdominant at high $P$, increasing $\Delta t$ increases the first error term in (6) and thus the overall error is larger. In this case, a higher-order backward integration method (higher $k$, e.g. Runge-Kutta of fourth-order) reduces the dominant first term and improves the solution.

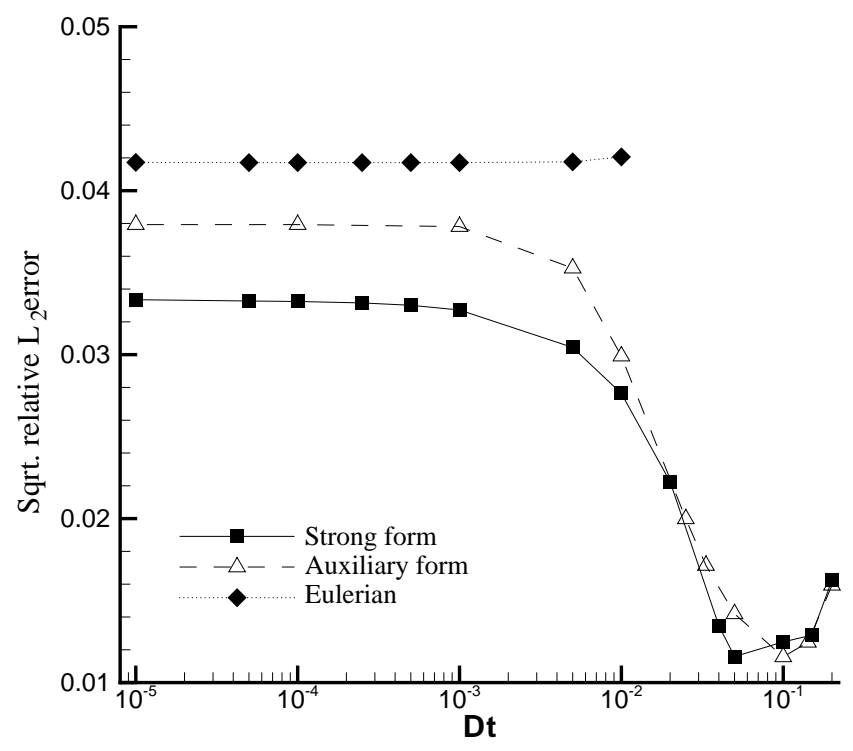

Figure 4: Error dependence upon $\Delta t$ with second-order integration for the strong and auxiliary semi-Lagrangian methods; also included is the Eulerian scheme. Errors are evaluated at a final time $T=1$ and at a polynomial order of $P=4$.

To further study the structure of the error of both the strong and auxiliary semi-Lagrangian methods, we test the SLSE method at different time steps and spectral order $P=4$. We set the viscosity to a small value, $\nu=4.6 \times 10^{-6}$, in order to emphasize the effect of the advection; the final time is $T=1$ here. In figure 4 we plot results obtained with second-order backward integration $(k=2)$ in semilog $x$-axes for the two semi-Lagrangian forms as well as the error corresponding to the Eulerian scheme. We see that for both semi-Lagrangian methods the error has the structure predicted theoretically. In particular, for this relatively low order $P=4$, the interpolation error is comparatively large, and thus the second error term in equation (6) dominates. As $\Delta t$ increases, the overall accuracy improves 
almost monotonically up to a large $\Delta t$ when the first error term becomes significant. For intermediate spectral orders (e.g. $P=6$ ), however, the interpolation error is smaller and the first error term in equation (6), $\mathcal{O}\left(\Delta t^{2}\right)$, is comparable with the second term. As $\Delta t$ increases, this error starts to decrease first. The $\mathcal{O}\left(\Delta t^{2}\right)$ term then becomes dominant and the overall error starts to increase. At this intermediate spatial resolution, there is clearly a competition between the two error terms resulting in the minimum error around $\Delta t \approx 0.04$. For higher spectral orders (e.g., $P=8$ ), the interpolation error is sufficiently small and thus the $\mathcal{O}\left(\Delta t^{2}\right)$ term dominates. The overall error then grows at an algebraic second-order rate. These observations are also based on the plots presented in [3]. In the auxiliary form, we recall that the solution of the auxiliary advection equation is required for $\tilde{\phi}$ which requires a CFL estimator is required to determine the number of substeps to be used for a specified $\Delta t$. At larger $\Delta t$ more substeps are therefore used, and so at the largest time steps the number of substeps had to be choosen manually. Due to the dominance of the error in the advection step for this problem we observe a plateau over a range of $\Delta t$ values.

\section{Incompressible Navier-Stokes Equations}

In this section we extend the semi-Lagrangian spectral element (SLSE) method to incompressible Navier-Stokes equations. We consider the Navier-Stokes equations in Lagrangian form

$$
\begin{gathered}
\frac{d \mathbf{u}}{d t}=-\nabla p+\nu \nabla^{2} \mathbf{u}, \\
\nabla \cdot \mathbf{u}=0,
\end{gathered}
$$

and present a second-order time-discretization scheme based on stiffly stable integration. In [3] it was shown that the Crank-Nicolson scheme is asymptotically unstable.

\subsection{Formulation}

A second-order stiffly-stable time-discretization gives

$$
\frac{\frac{3}{2} \mathbf{u}^{n+1}-2 \mathbf{u}_{d}^{n}+\frac{1}{2} \mathbf{u}_{d}^{n-1}}{\Delta t}=\left(-\nabla p+\nu \nabla^{2} \mathbf{u}\right)^{n+1}
$$

where $\mathbf{u}_{d}^{n}$ is the velocity $\mathbf{u}$ at the departure point $\mathbf{x}_{d}^{n}$ at time level $t^{n}$ and $\mathbf{u}_{d}^{n-1}$ is the velocity at the departure point $\mathbf{x}_{d}^{n-1}$ at time level $t^{n-1}$. In the strong semi-Lagrangian form the departure points $\mathbf{x}_{d}^{n}$ and $\mathbf{x}_{d}^{n-1}$ are obtained by solving the characteristic equation over a single time level $\Delta t$ and over two time levels $2 \Delta t$, respectively. In the auxiliary semi-Lagrangian method the auxiliary advection equation is solved to obtain $\mathbf{u}_{d}^{n-1}$ and $\mathbf{u}_{d}^{n}$ directly. 
A three-step splitting scheme can be used to solve (9), i.e.,

$$
\begin{aligned}
\frac{\hat{\mathbf{u}}-2 \mathbf{u}_{d}^{n}+\frac{1}{2} \mathbf{u}_{d}^{n-1}}{\Delta t} & =0, \\
\frac{\hat{\mathbf{u}}-\hat{\mathbf{u}}}{\Delta t} & =-\nabla p^{n+1}, \\
\frac{\frac{3}{2} \mathbf{u}^{n+1}-\hat{\mathbf{u}}}{\Delta t} & =\nu \nabla^{2} \mathbf{u}^{n+1} .
\end{aligned}
$$

The discrete divergence-free condition $\nabla \cdot \mathbf{u}^{n+1}=0$ results in a consistent Poisson equation for the pressure

$$
\nabla^{2} p^{n+1}=\frac{1}{\Delta t} \nabla \cdot \hat{\mathbf{u}}
$$

with accurate pressure boundary conditions of the form [8]

$$
\frac{\partial p}{\partial n}=-\mathbf{n} \cdot\left[\hat{\mathbf{u}} / \Delta t+\nu \nabla \times \omega^{n}\right]
$$

where $\mathbf{n}$ is the unit normal, we assume that $u^{n+1}=0$ on the boundary and $\omega$ is the vorticity. Although the pressure boundary condition is treated explicitly, the overall scheme is unconditionally stable as has shown rigorously in [27] for the Stokes problem for first- and second-order schemes.

\subsection{Numerical Results}

In this section we present numerical results by applying the above discretizations to different benchmark problems. In all the tests employing the strong SLSE method, the second-order Runge-Kutta method is employed for backward integration.

First, we use the Taylor vortex problem, an exact solution to the unsteady Navier-Stokes equations, in order to quantify the error in the SLSE method. It has the form

$$
\begin{aligned}
u & =-\cos x \sin y e^{-2 t / R e}, \\
v & =\sin x \cos y e^{-2 t / R e}, \\
p & =-\frac{1}{4}(\cos 2 x+\cos 2 y) e^{-4 t / R e} .
\end{aligned}
$$

The computational domain is a square defined by the coordinates $\left[-\frac{\pi}{2}, \frac{\pi}{2}\right]$ in each direction. A mesh consisting of $2 \times 2$ quadrilateral elements is used and the Reynolds number is fixed at $10^{6}$.

In figure 5(left) we plot the dependence of the error upon the size of time step for the stiffly-stable method at a final time of $T=2 \pi$. The results are similar to the behavior reported earlier for the advection-diffusion equation. Note here the accuracy of SLSE method is dictated by the term $\mathcal{O}\left(\Delta t^{2}+\frac{\Delta x^{P+1}}{\Delta t}\right)$. At low $P$ the interpolation error 

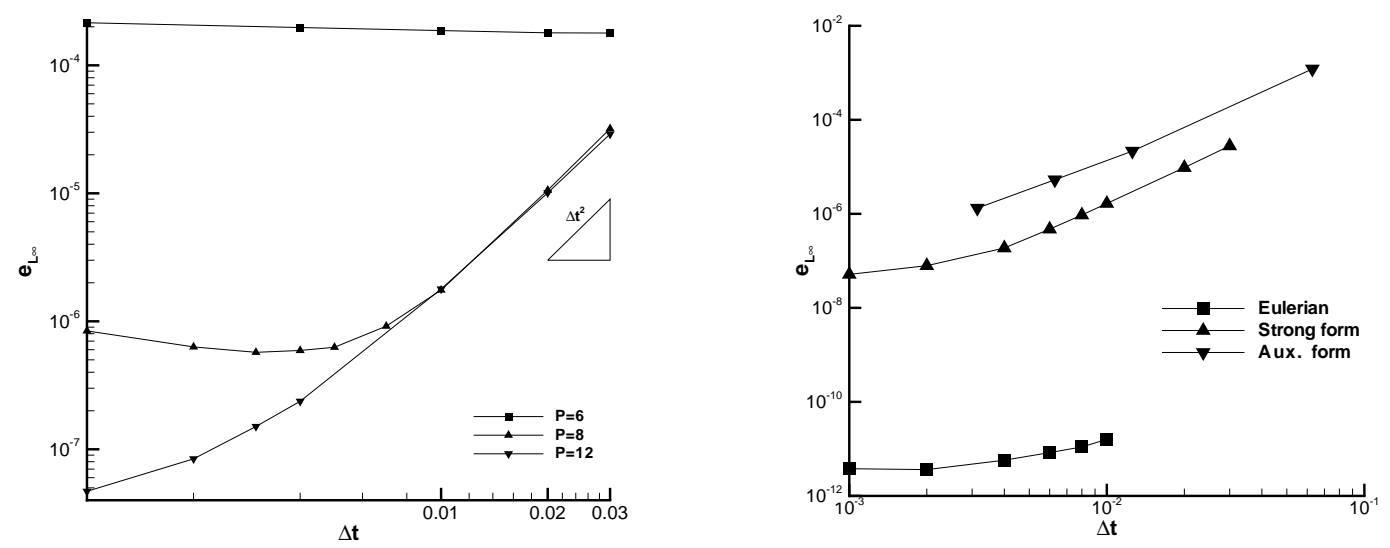

Figure 5: Error dependence on $\Delta t$. Left: strong SLSE results, and Right: comparison of strong and auxiliary SLSE for $P=12$.

dominates, and increasing $\Delta t$ decreases the overall error, as shown for $P=6$. When the interpolation error is small at $P=12$, the $\Delta t^{2}$ term dominates and further increase in $\Delta t$ increases the overall error. The $P=8$ curve shows the competition between these two terms. In this plot, the largest time step 0.03 corresponds to the CFL number of about 4. In figure 5(right) we compare the strong and auxiliary SLSE at polynomial order $P=12$. Both SLSE schemes demonstrate a similar error dependence with $\Delta t$ whereas the Eulerian scheme has a much smaller error. Recalling the high Reynolds number adopted in this test we observe that the solution over the time period considered is close to being constant. The Eulerian scheme therefore benefits from the more stationary nature of the solution since there is no time error associated with this Eulerian scheme for an exactly stationary problem. We emphasize that for both the SLSE schemes it is the size of $\Delta t$ and not the CFL number that restricts the use of semi-Lagrangian method. In other words, for the SLSE method, the restriction on the size of time step is solely due to accuracy considerations but not due to stability. Finally, we note that in the auxiliary semi-Lagrangian method a mixed second-order RungeKutta and Adams-Bashforth scheme was applied in time to solve the auxiliary advection equation. In this approach the first step of the auxiliary problem is advanced using a second-order Runge-Kutta schmee. In subsequent steps a second order Adams-Bashforth scheme is applied, which is computationally more efficient due to the multi-step nature since it requires fewer right-hand-side evaluations than the Runge-Kutta schemes per time step.

The extension of the SLSE method to three dimensions is straight-forward. Here we study the effect of the threedimensionality in the driven cavity flow as a function of the aspect ratio. The Reynolds number is set at 400 and the aspect ratio was set to 1,3 and 5 ; the spectral order is $P=10$. We plot velocity profiles at the center symmetric plane in figure 6 . Three-dimensionality effects are more pronounced, as expected, for the expansion ratio 1 as the 

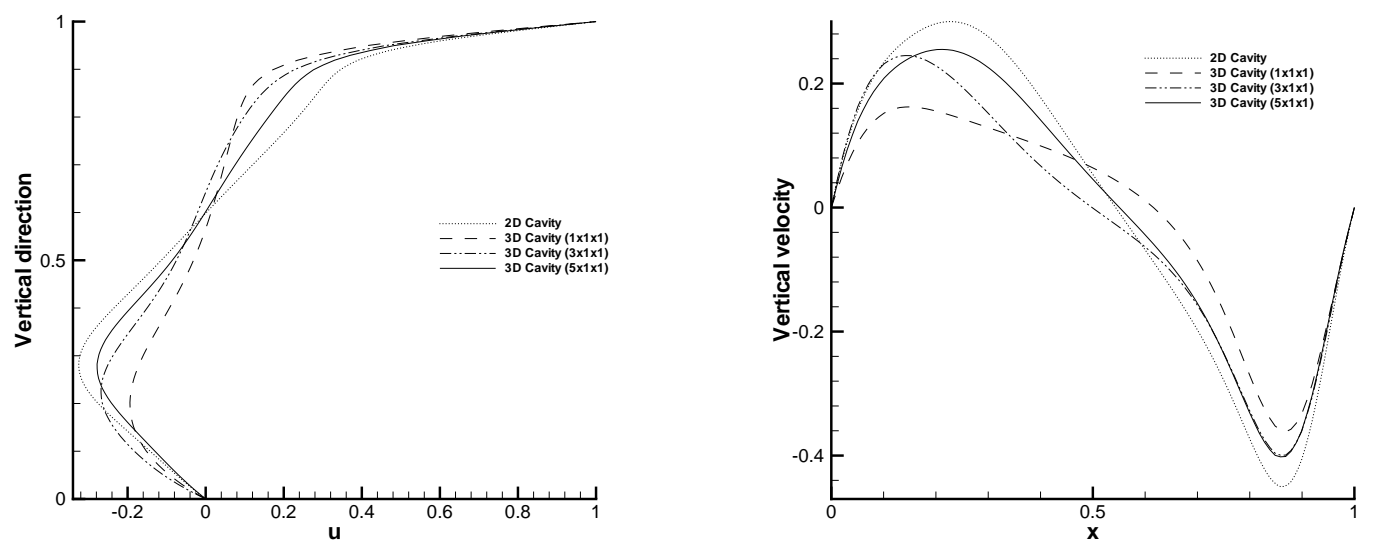

Figure 6: 3D driven cavity flow at $R e=400$. Left: horizontal-velocity profile along vertical centerline, Right: vertical-velocity profile along horizontal centerline.

results deviate the most from the corresponding two-dimensional profile, while for the aspect ratio 5 , a trend towards two-dimensionality is observed. The results shown in the figure are obtained by SLSE method with CFL number 20. Results of the Eulerian spectral/hp element method are also computed with CFL number at 0.6 but are not shown in the plot because they are essentially identical as the SLSE results. In [21], the semi-Lagrangian (quadratic) finite element method is used to solve the $2 \mathrm{D}$ driven cavity flow. The reported results show significant difference with results of [28], and the authors claim favorable comparison with three-dimensional experimental results of [29]. The present simulations of the three-dimensional driven cavity flow suggest that such an agreement may have been fortuitous. A possible explanation for such large discrepancy is an incorrect treatment of pressure in the formulation of [21].

\subsection{Hybrid Discretizations}

In turbulent channel flow simulations Fourier expansions are preferrable in the homogeneous directions; in the wall-normal direction spectral/hp element discretization can be employed. When one applies the semi-Lagrangian method to channel simulations with such a hybrid discretization, Lagrange interpolation is the natural choice for interpolations in the wall-normal direction, which is consistent with the spectral/hp elment discretizations [8]. In the homogeneous directions, the most straight-forward interpolation approach is to construct the interpolant employing all the Fourier modes. However, the computional cost with this approach turns out to be very high [30]. We present next two local high-order interpolation schemes in the Fourier directions for the semi-Lagrangian method.

We employ Lagrange and Hermite local interpolations in Fourier directions in the strong semi-Lagrangian method. Given a set of distinct points $\left\{x_{j}\right\}_{j=0}^{n}$, some function $f(x)$ and its derivative $f^{\prime}(x)$ on these points, the $(2 n+1)$ degree 


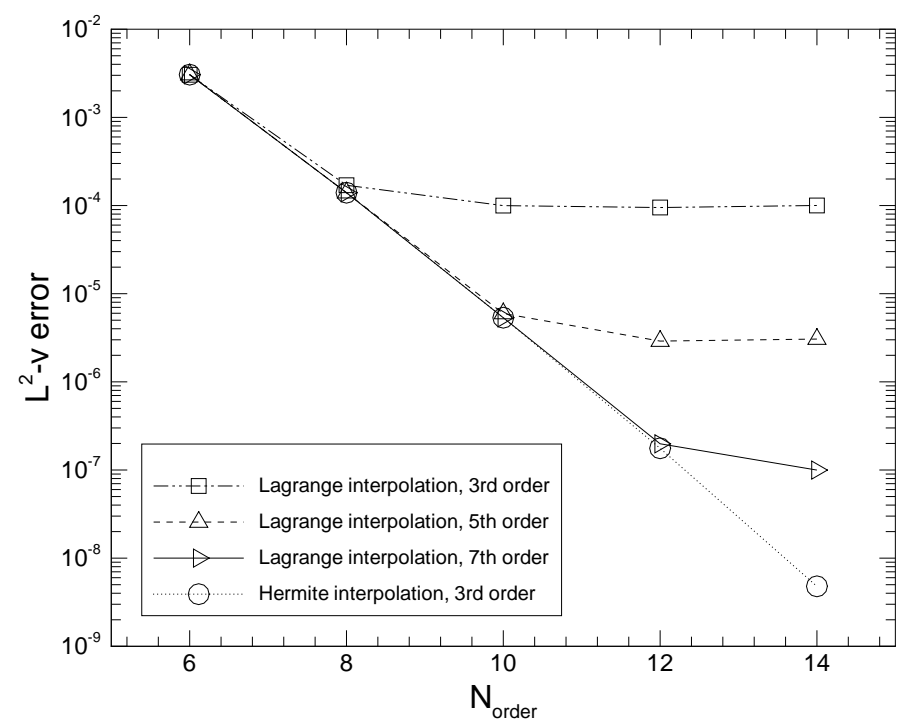

Figure 7: $L^{2}$ error of $y$-component velocity as a function of the polynomial order of the spectral element, $P$, for an $3 \mathrm{D}$ unsteady analytic flow field.

Hermite interpolant approximating the function is expressed by

$$
p(x)=\sum_{j=0}^{n} f\left(x_{j}\right) H_{n, j}(x)+\sum_{j=0}^{n} f^{\prime}\left(x_{j}\right) \hat{H}_{n, j}(x),
$$

where

$$
H_{n, j}(x)=\left[1-2\left(x-x_{j}\right) L_{n, j}^{\prime}\left(x_{j}\right)\right] L_{n, j}^{2}(x), \quad \hat{H}_{n, j}(x)=\left(x-x_{j}\right) L_{n, j}^{2}(x),
$$

and $L_{n, j}(x)$ is the Lagrange polynomial

$$
L_{n, j}(x)=\prod_{i=0, i \neq j}^{n} \frac{x-x_{i}}{x_{j}-x_{i}} .
$$

Let us assume that $x$ and $z$ are the homogeneous directions of the channel. The velocity derivatives $\frac{\partial u}{\partial x}, \frac{\partial u}{\partial z}$ and $\frac{\partial^{2} u}{\partial x \partial z}$, on the grid points need to be computed when the Hermite interpolation is applied in the semi-Lagrangian method. We can obtain these derivatives accurately and efficiently by computing them in the Fourier space followed by an inverse Fourier transform.

We examine the accuracy of the semi-Lagrangian method with Lagrange and Hermite local interpolations with a 3D analytic flow solutions to the unsteady Navier-Stokes equations expressed by

$$
\begin{aligned}
u & =\sin (m x) \cos (l y) \cos (n z) e^{-t / R e}, \\
v & =-\frac{m+n}{l} \cos (m x) \sin (l y) \cos (n z) e^{-t / R e}, \\
w & =\cos (m x) \cos (l y) \sin (n z) e^{-t / R e},
\end{aligned}
$$




$$
\begin{aligned}
p= & -\frac{m+n}{l^{2} R e}\left(m^{2}+l^{2}+n^{2}-1\right) \cos (m x) \cos (l y) \cos (n z) e^{-t / R e} \\
& +\left[\frac{m(m+n)}{4 l^{2}} \sin ^{2}(m x) \cos (2 l y) \cos ^{2}(n z)\right. \\
& +\frac{(m+n)^{2}}{4 l^{2}} \cos ^{2}(m x) \cos (2 l y) \cos ^{2}(n z) \\
& \left.+\frac{n(m+n)}{4 l^{2}} \cos ^{2}(m x) \cos (2 l y) \sin ^{2}(n z)\right] e^{-2 t / R e},
\end{aligned}
$$

subject to the following force

$$
\begin{aligned}
f_{x}= & \frac{1}{R e}\left(m^{2}+l^{2}+n^{2}-1\right)\left[1+\frac{m(m+n)}{l^{2}}\right] \sin (m x) \cos (l y) \cos (n z) e^{-t / R e} \\
& +(m / 2) \sin (2 m x) \cos ^{2}(l y) \cos ^{2}(n z) e^{-2 t / R e} \\
& +((m+n) / 2) \sin (2 m x) \sin ^{2}(l y) \cos ^{2}(n z) e^{-2 t / R e} \\
& -(n / 2) \sin (2 m x) \cos ^{2}(l y) \sin ^{2}(n z) e^{-2 t / R e} \\
& -\frac{m n(m+n)}{4 l^{2}} \sin (2 m x) \cos (2 l y) e^{-2 t / R e}, \\
f_{y}= & 0, \\
f_{z}= & \frac{1}{R e}\left(m^{2}+l^{2}+n^{2}-1\right)\left[1+\frac{n(m+n)}{l^{2}}\right] \cos (m x) \cos (l y) \sin (n z) e^{-t / R e} \\
& -(m / 2) \sin ^{2}(m x) \cos ^{2}(l y) \sin (2 n z) e^{-2 t / R e} \\
& +((m+n) / 2) \cos ^{2}(m x) \sin { }^{2}(l y) \sin (2 n z) e^{-2 t / R e} \\
& +(n / 2) \cos ^{2}(m x) \cos ^{2}(l y) \sin (2 n z) e^{-2 t / R e} \\
& -\frac{m n(m+n)}{4 l^{2}} \cos ^{2}(2 l y) \sin (2 n z) e^{-2 t / R e} .
\end{aligned}
$$

The flow domain is a $(2 \pi)^{3}$ cube $(0 \leq x \leq 2 \pi,-\pi \leq y \leq \pi, 0 \leq z \leq 2 \pi)$. The homogeneous directions are $x$ and $z$ directions, in which periodic boundary conditions are applied. Dirichlet boundary conditions based on the analytic solution are imposed at $y=-\pi, \pi$. We employ 16 grid points in $x$ and $z$ directions and one spectral element in the $y$ direction. The Navier-Stokes equations are integrated with a stiffly-stable semi-Lagrangian method (second-order accurate in time) from $t=0$ to $t=T$. We then compute the $L^{2}$ error of the computed flow field at $t=T$ against the exact solution. We employ Lagrange interpolation and Hermite interpolation for interpolating the velocity on the departure points, respectively. The number of grid points is fixed in homogensous directions, and the order of the spectral element in the $y$ direction is varied in the tests. Figure 7 shows the $L^{2}$ error of the $y$ velocity component as a function of the spectral element order for a flow with the following parameters: $m=l=n=1, R e=1.0, T=1.0$. With Hermite interpolation we observe exponential decrease of the error whereas with Lagrange interpolation we only observe exponential decrease of the error at low element orders; this trend disappears at high element orders for a fixed 


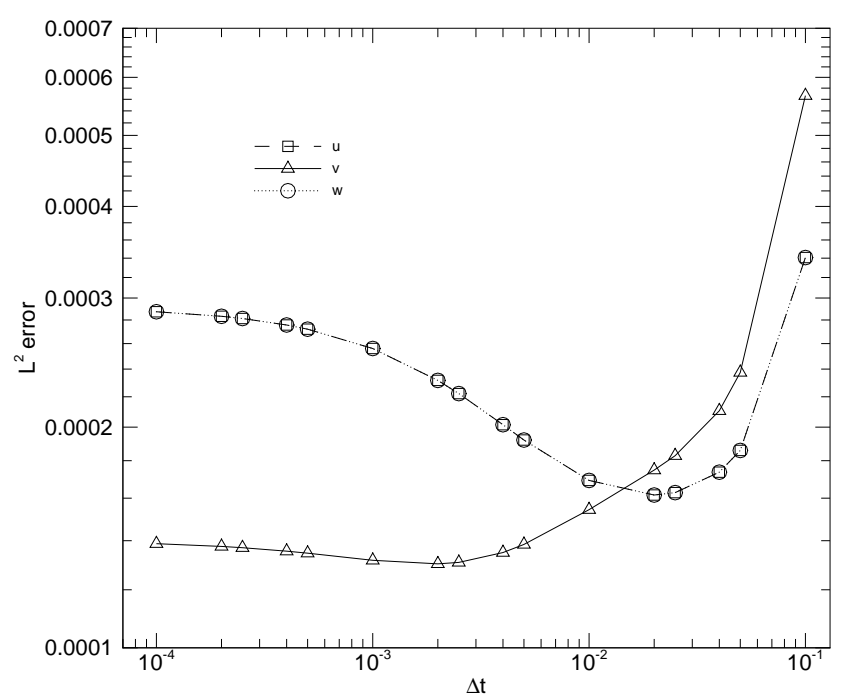

Figure 8: $L^{2}$ error as function of time step width $\Delta t$. The order of the spectral element is 8 in $y$-direction.

order of Lagrange interpolation. We also observe that for increasing order of Lagrange interpolation the exponential convergence continues. However, increasing the Lagrange interpolation order notably increases the computational cost. For example, numerical experiments show that a seventh-order Lagrange interpolation computation costs about twice as much as a third-order Hermite interpolation computation for this test problem. These results indicate that for the semi-Lagrangian method, Hermite interpolation is superior in accuracy and cost-effectiveness to the Lagrange interpolation. Next we fix the order of the spectral element at $P=8$, and then vary the time step width $\Delta t$. In figure 8 we plot the $L^{2}$ errors of the velocity as a function of the time step with Hermite interpolation. The results demonstrate a trend of error consistent with that expressed by equation (6).

Interpolations are the most expensive operations in the semi-Lagrangian method. The local nature of Hermite and Lagrange interpolations and the uniform grid in the homogeneous directions allow for an efficient parallelization scheme for handling interpolations in this method. The channel flow domain is decomposed in the streamwise direction, with each processor computing one sub-domain. In the interpolation step, we differentiate grid points in a "shadow region" near the boundary of each sub-domain from those points in the interior of the sub-domain. Interpolation on the points in the shadow region depends on the velocity data from neighboring processors. Interpolation on the interior points requires data from the same processor only. Therefore, only data in the shadow regions need to be communicated between neighboring processors in the interpolation step. Parallel efficiency can be greatly improved by overlapping the communications of the shadow data with the computations on the interior points with non-blocking message-passing routines such as those provided by the Message Passing Interface (MPI). Specifically, 


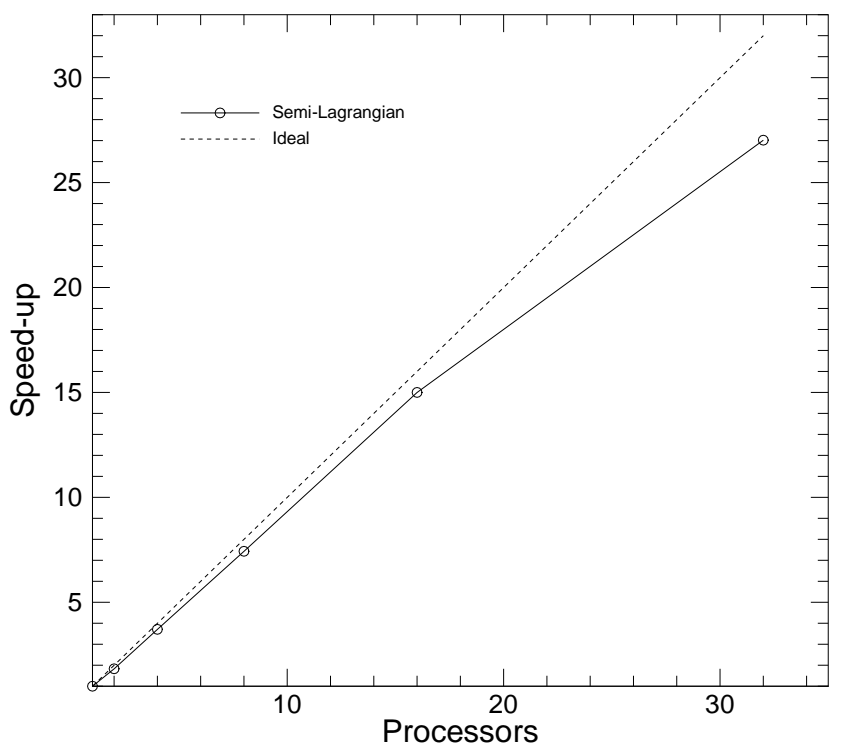

Figure 9: Parallel speedup of the strong semi-Lagrangian method with Hermite interpolation on a grid $64^{3}$ for a turbulence channel simulation. One spectral element of order $P=64$ was used in the $y$-direction.

before interpolating on the interior points each processor first posts non-blocking send/receive requests with neighboring processors. Then, all processors perform interpolations on their own interior points, while communications of the shadow data are handled by the system in the background. After completing the interior interpolation, the processor checks the status of non-blocking communications, which is most likely already complete. So the processor proceeds to interpolate on the shadow points. In rare cases when the comunication is still not complete, the processor needs to wait until the shadow data becomes available. With the above method, we can effectively hide communications of the shadow data behind the useful computations on the interior points. Figure 9 shows the parallel speedup of the semi-Lagrangian method with Hermite interpolation on a $64^{3}$ grid, demonstrating a good parallel efficiency.

\section{Summary and Discussion}

Two forms of the semi-Lagrangian method discretized with high-order in space were presented and applied to advection-diffusion and incompressible Navier-Stokes equations. The strong form is free from the CFL-restriction and thus very large time steps can be used, dictated only by accuracy considerations. This should not be interpreted as violation of the CFL condition, as the numerical domain of dependence of the solution still contains its domain of dependence [9]. The auxiliary form extends substantially the CFL number compared to the Eulerian scheme but it is not CFL-free because of the explicit treatment of an auxiliary advection equation in a substepping procedure. 
An interesting aspect of the method is the structure of the temporal (advection) error, which reveals non-monotonic trend with the time step. This behavior depends strongly on the interpolation procedure involved and inaccurate representations may mask this trend.

With regards to efficiency, both the strong and auxiliary semi-Lagrangian methods require more computational cost than the Eulerian counterpart on a per time step consideration. In the strong semi-Lagrangian method we require backwards particle tracking from every quadrature point and then interpolation of the polynomial approximation at non-quadrature points. Backward particle tracking can be expensive in deformed elements and is more intricate to parallelize. In contrast, the auxiliary semi-Lagrangian method uses "more standard" implementation from the Eulerian point of view and so can benefit from existing concepts for parallelisation. However, the cost of performing each full time step $\Delta t$ in the auxiliary approach is dependent upon the number of substeps that are necessary to maintain the CFL stability of the advection problem. Therefore, if the cost of solving one step of the advection problem is the same as the cost of inverting the implicit diffusion operator in the previous advection-diffusion example then there would not appear to be any benefit of using the auxiliary semi-Lagrangian method independent of the size of $\Delta t$ that would be achieved. The ratio of the computational cost of the advection step to the diffusion step is therefore very important. However, for the strong semi-Lagrangian method there is a distinct advantage to using larger time steps providing stability restrictions are not violated. This is because the cost of the backward particle tracking in the strong semi-Lagrangian method is relatively independent of $\Delta t$. It would therefore seem that for intermediate time steps the auxiliary semi-Lagrangian method is preferrable whereas for large time steps the strong semi-Lagrangian will prevail.

We now turn our attention to the stability of the strong semi-Lagrangian method, which has been studied in [9]. In particular, $L^{\infty}$ stability holds for any low-order interpolation, i.e., piecewise linear or bilinear approximation. In the general linear high-order case, unconditional stability with respect to time step holds for fixed spatial discretization for any $\Delta t>0$. However, this is not true with respect to any order of interpolation, say order $P$, unless monotone interpolating schemes are used for the departure point.

With respect to the $L^{2}$ stability, using von Neumann analysis, Falcone \& Ferretti [9] showed that for equidistant interpolations of order higher than second instabilities may arise on a fixed grid. However, this can be overcome by employing smaller "sliding" stencils of grid points surrounding the departure point $\mathbf{x}_{d}$. This is what is actually done in practice. For non-equidistant grids with Lagrangian or Hermite interpolations, $L^{2}$ stability holds although a rigorous theory is incomplete.

The question of consistency of semi-Lagrangian schemes has also been raised in the past. We note, however, that 
as $\Delta t, \Delta x \rightarrow 0$, then it is easy to show that the error also aproaches zero. For finite $\Delta x$ and $\Delta t \rightarrow 0$, we see from figure 4 that the error goes to a constant value dictated by the spatial discretization error.

Another cause of instability may be due to the intersection of the approximate trajectories. A sufficient condition to avoid this has been derived in [31] and states that

$$
\Delta t<\left|\mathbf{J}_{a}^{-1}\right|
$$

involving the Jacobian with respect to the velocity field a, which in general varies in space and time.

Finally, boundary conditions should be carefully treated as the tracking procedure may search for points outside the domain due to the large time step taken. In [9], a modified algorithm is proposed, where the grid points around the boundary are treated with a timestep $\delta t<\Delta t$. This clearly complicates the implementation.

\section{Acknowledgements}

We would like to thank Dr. F.X. Giraldo for providing some of his results. This work was supported partially by ONR, DOE and NSF. The computations were performed on DoD's MSRC computers (NAVO, ERDC and ARSC).

\section{References}

[1] R.D. Loft, S.J. Thomas, and J.M. Dennis. Terascale spectral element dynamical code for atmospheric general circulation models. In Proceedings of Supercomuting 2001, Denver, 2001.

[2] Y. Maday, A.T. Patera, and E.M. Ronquist. An operator-integration-factor splitting method for time-dependent problems: Application to incompressible fluid flow. J. Sci. Comp., 4:263-292, 1990.

[3] D. Xiu and G.E. Karniadakis. A semi-Lagrangian high-order method for Navier-Stokes equations. J. Comp. Phys., 172:658, 2001.

[4] A. Robert. A stable numerical integration scheme for the primitive meteorological equations. Atmos. Ocean, 19:35, 1981.

[5] O. Pironneau. On the transport-diffusion algorithm and its applications to the Navier-Stokes equations. Numer. Math., 38:309, 1982 .

[6] F.X. Giraldo. Strong and weak Lagrange-Galerkin spectral element methods for shallow water equations. Computers and Mathematics with Applications, 45:97-121, 2003. 
[7] G.E. Karniadakis and S.A. Orszag. Nodes, modes, and flow codes. Phys. Today, 34, 1993.

[8] G.E. Karniadakis and S.J. Sherwin. Spectral/hp element methods for CFD. Oxford University Press, London, 1999.

[9] M. Falcone and R. Ferretti. Convergence analysis for a class of high-order semi-Lagrangian advection schemes. SIAM J. Numer. Anal., 35:909, 1998.

[10] J.P. Huffenus and D. Khaletzky. A finite element method to solve the Navier-Stokes equations using the method of characteristics. Int. J. Numer. Methods Fluids, 4:247, 1984.

[11] A.V. Malevsky and S.J. Thomas. Parallel algorithms for semi-Lagrangian advection. Int. J. Numer. Methods Fluids, 25:455, 1997.

[12] A. Oliveira and A.M. Baptista. A comparison of integration and interpolation Eulerian-Lagrangian methods. Int. J. Numer. Methods Fluids, 21:183, 1995.

[13] A. Staniforth and J. Cote. Semi-Lagrangian integration schemes for atmospheric models - a review. Mon. Wea. Rev., 119:2206, 1991.

[14] A.V. Malevsky. Spline-characteristic method for simulation of convective turbulence. J. Comput. Phys., 123:466, 1996.

[15] P. Bartello and S.J. Thomas. The cost-effectiveness of semi-Lagrangian advection. Mon. Wea. Rev., 124:2883, 1996.

[16] F.X. Giraldo. The Lagrange-Galerkin spectral element method on unstructured quadrilateral grids. J. Comput. Phys., 147:114, 1998.

[17] J.L. McGregor. Economical determination of departure points for semi-Lagrangian models. Mon. Wea. Rev., 121:221, 1993.

[18] A. McDonald and J.R. Bates. Improving the estimate of the departure point position in a two-time level semi-Lagrangian and semi-implicit scheme. Mon. Wea. Rev., 115:737, 1987.

[19] A. McDonald. Accuracy of multi-upstream, semi-Lagrangian advective schemes. Mon. Wea. rev., 112:1267, 1984. 
[20] E.S. Suli. Convergence and nonlinear stability of the Lagrange-Galerkin method for the Navier-Stokes equations. Numer. Math., 53:459, 1988.

[21] A. Allievi and R. Bermejo. Finite element modified method of characteristics for the Navier-Stokes equations. Int. J. Numer. Methods Fluids, 32:439, 2000.

[22] A. Priestley. Exact projections and the Lagrange-Galerkin method: A realistic alternative to quadrature. $J$. Comput. Phys., 112:316, 1994.

[23] Y. Achdou and J.L. Guermond. Convergence analysis of a finite element projection/Lagrange-Galerkin method for the incompressible Navier-Stokes equations. SIAM J. Numer. Anal., 37:799, 2000.

[24] M.O. Deville, P.F. Fischer, and E.H. Mund. High-Order Methods for Incompressible Fluid Flow. Cambridge University Press, 2002.

[25] S.H. Momeni-Masuleh. Spectral Methods for the Three Field Formulation of Incompressible Fluid Flow. PhD thesis, Aberystwyth, 2001.

[26] S.J. Sherwin. A substepping Navier-Stokes splitting scheme for spectral/ $h p$ element discretisations. In T. Matuson, A. Ecer, J. Periaux, N. Satufka, and P. F ox, editors, Parallel Computational Fluid Dynamics: New Fonrtiers and Multi -Disciplinary Applications, pages 43-52. North-Holland, 2003.

[27] E. Leriche and G. Labrosse. High-order direct Stokes solvers with or without temporal splitting: Numerical investigations of their comparative properties. SIAM J. Sci. Comput., 22(4):1386, 2000.

[28] U. Ghia, K.N. Ghia, and C.T. Shin. High-Re solutions for the incompressible flow using the Navier-Stokes equations and a multigrid method. J. Comput. Phys., 48:387, 1982.

[29] J.R. Koseff and R.L. Street. The lid-driven cavity flow: a synthesis of qualitative and quantitative observations. ASME J. Fluids Eng., 106:390, 1984.

[30] J. Xu, D. Xiu, and G.E. Karniadakis. A semi-Lagrangian method for turbulence simulations using mixed spectral discretizations. J. Sci. Comput., 17:585, 2002.

[31] P.K. Smolarkiewicz and J. Pudykiewicz. A class of semi-Lagrangian approximations for fluids. J. Atmo. Sci., 49:2082, 1992. 